\title{
Modelling vocabulary acquisition, adaptation and generalization in infants using adaptive Bayesian PLSA
}

\author{
J. Driesen *, H. Van hamme \\ Department ESAT, K.U.Leuven, Leuven, Belgium
}

\section{A R T I C L E I N F O}

Available online 24 February 2011

\section{Keywords:}

NMF

PLSA

Vocabulary acquisition

Learning

Memory

\begin{abstract}
A B S T R A C T
During the early stages of language acquisition, young infants face the task of learning a basic vocabulary without the aid of prior linguistic knowledge. Attempts have been made to model this complex behaviour computationally, using a variety of machine learning algorithms, a.o. non-negative matrix factorization (NMF). In this paper, we replace NMF in a vocabulary learning setting with a conceptually similar algorithm, probabilistic latent semantic analysis (PLSA), which can learn word representations incrementally by Bayesian updating. We further show that this learning framework is capable of modelling certain cognitive behaviours, e.g. forgetting, in a simple way.
\end{abstract}

(c) 2011 Elsevier B.V. All rights reserved.

\section{Introduction}

Understanding speech is one of the most complex tasks the human brain is capable of. Effortlessly, it induces the meaning from an uninterrupted stream of sound using a vast amount of knowledge: vocabulary, acoustic models, syntax, etc. The robustness and accuracy that is achieved in this process has not been matched by any known computational technique to date. Children start developing these language skills almost from the moment they are born. At this point they have no knowledge of speech whatsoever. All they perceive from speech is a continuous stream of auditory stimuli. The first challenge a newly born faces is therefore to detect word boundaries within this stream, i.e. to "crack the speech code", so they can proceed by learning to recognize the word-like patterns enclosed between them (see $[4,6,7]$ ). It has been shown experimentally that children use statistics of acoustic transitions to achieve this goal (see $[5,24]$ ). Sounds that often co-occur are gradually categorized as belonging to the same word-like pattern, whereas relatively rare co-occurrences most likely indicate a boundary between patterns. The meaning of these word-like patterns is derived from other modalities than speech. If a sound pattern is perceived often enough in conjunction with a certain concept in another modality, e.g. a baby often hears the pattern "daddy" upon seeing its father, a semantic link is established between them (see e.g. [22]). When this happens, a "word" is added to the child's vocabulary. This limited vocabulary then becomes the basis for more complex

\footnotetext{
* Corresponding author.

E-mail addresses: joris.driesen@esat.kuleuven.be (J. Driesen), hugo.vanhamme@esat.kuleuven.be (H. Van hamme).
}

linguistic analyses. A computational model for exactly this process will be discussed in this paper.

A wide array of machine learning techniques has been considered to model language acquisition computationally. Almost invariably, they use multi-modal inputs in which they detect patterns within the speech modality that are then statistically related to the concepts in the other modalities. This process is referred to in literature as "grounding". One of those learning techniques that has recently been proposed is non-negative matrix factorization (NMF) (see [8]), an algorithm that has already proven to be quite successful in the area of data mining, a.o. because of its attractive computational properties and its relative simplicity. For modelling language acquisition, it is interesting because it is able to find structure in speech without any prior linguistic knowledge, by making use of statistical regularities and irregularities within the speech signal, similar to what real infants do (see $[27,28,23]$ ).

However, NMF is a batch algorithm that processes hours of speech simultaneously to extract a number of word-like patterns at once. From a cognitive perspective, it is hard to justify that this algorithm models the language learning behaviour of a child, in which the internal word representations seem to evolve gradually over time (see e.g. [3]). Moreover, modelling a complex behaviour like language acquisition with a batch algorithm implies the assumption that an infant uses all stimuli ever perceived and remembered in a similar fashion and with the same level of detail. No distinction is made between recently perceived data and data that was perceived long ago, even though the latter is more likely to be inaccurate, due to the fallibility of human memory.

In this paper, we address these shortcomings of NMF. We consider a NMF-based framework for vocabulary acquisition and replace the learning algorithm by a conceptually similar one called probabilistic latent semantic analysis (PLSA), proposed 
in [26]. This algorithm is capable of adaptive learning by performing Bayesian re-estimation of its internal models as was shown in [2]. In Section 2 of this paper, we describe NMF and how it relates to PLSA. In Section 3, we discuss how PLSA should be made adaptive in the context of word learning. In Section 5, we clarify how keyword recognition can be performed by factorizing a data matrix. In Section 6 we show some experimental results followed by a discussion in Section 7. We finish with some concluding remarks in Section 8.

\section{Structure discovery by NMF}

NMF is a factorization algorithm that decomposes a data matrix $\mathbf{V}$, of size $M \times N$, into the product of two matrices $\mathbf{W}$ and $\mathbf{H}$, of size $M \times R$ and $R \times N$, respectively. Typically, $R$ is small compared to $M$ and $N$.

$\mathbf{V} \approx \mathbf{W} \cdot \mathbf{H}$

This factorization can be solved by minimizing the Kullback-Leibler divergence between $\mathbf{V}$ and $\mathbf{W} \cdot \mathbf{H}$ :

$D_{K L}(\mathbf{V} \| \mathbf{W H})=\sum_{i j}\left(V_{i j} \log \frac{V_{i j}}{(W H)_{i j}}+(W H)_{i j}-V_{i j}\right)$

which can be done by iteratively applying the multiplicative update formulas for $T$ iterations (see [8]):

for I iterations do

$$
W_{i k} \leftarrow W_{i k} \cdot \sum_{j} H_{k j}\left(\frac{V}{W H}\right)_{i j}
$$

normalize : $\sum_{i} W_{i k}=1$

$H_{k j} \leftarrow H_{k j} \cdot \sum_{i} W_{i k}\left(\frac{V}{W H}\right)_{i j}$

What sets this NMF apart from other matrix factorizations such as principal component analysis or eigenvalue decomposition, is that $\mathbf{V}, \mathbf{W}$ and $\mathbf{H}$ only contain non-negative elements. This means that each column of $\mathbf{V}$ can be approximated by a weighted sum of the columns in $\mathbf{W}$, where the weights are the elements of the corresponding column in $\mathbf{H}$. A hypothetical example is shown in Fig. 1, where a vectorized representation of a bike $\mathbf{V}_{\mathbf{j}}$ is decomposed into vectorized representations of its parts, contained in the columns of $\mathbf{W}$. The weights of these parts are then the elements of $\mathbf{H}_{\mathbf{j}}$. A more practical example is shown in Fig. 2. If spoken utterances can be represented as non-negative vectors, NMF will find patterns that ideally correspond to words. An example of characterizing speech in this way is the so-called histogram of acoustic co-occurrences, or HAC, which will be described below, in Section 5.2.

It is implicitly assumed here that NMF automatically finds solutions where the columns of $\mathbf{W}$ contain localized parts of the data, allowing us to interpret them physically as "building

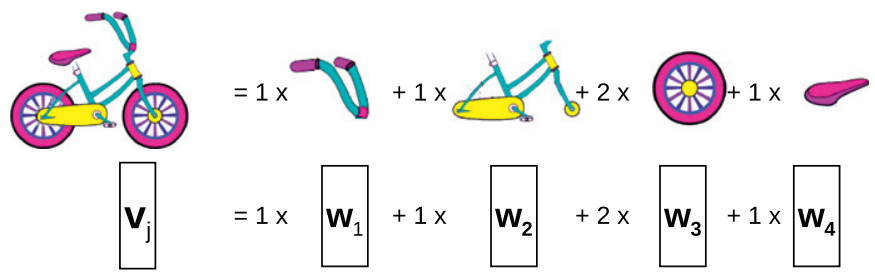

Fig. 1. An example of a parts-based decomposition. The representation of a complete bike by a vector $\mathbf{V}_{\mathbf{j}}$ is decomposed into a weighted sum of the vector representations of its constituent parts $\mathbf{W}_{\mathbf{i}}$.

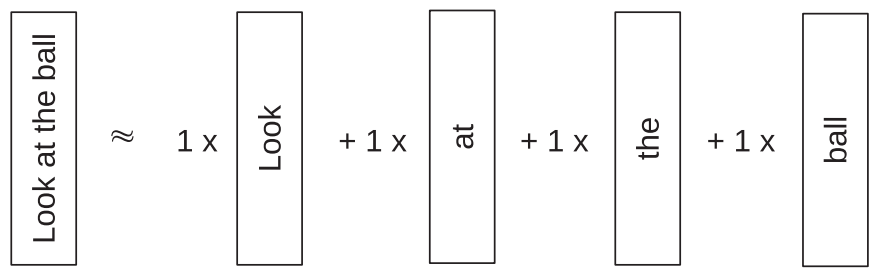

Fig. 2. Parts-based decomposition applied on speech. A vector representing a spoken utterance is decomposed into a weighted sum of the vector representations of its constituent parts, i.e. word-like patterns.

blocks". Such solutions, while possible, are not guaranteed by the NMF algorithm as it is presented above. Additional constraints are usually necessary to enforce them (see $[30,1,31])$. However, as will be discussed in Section 5, the learning framework in this paper uses supervised training in such a way that any word-like pattern that corresponds to a vocabulary entry is assigned to a single column of $\mathbf{W}$. The necessity of additional constraints to find a solution that suits our purposes (i.e. vocabulary acquisition) is therefore greatly diminished in this context.

\subsection{Link with PLSA}

NMF is closely linked to PLSA (see [26]). PLSA is a method that attempts to find a statistical variable $\mathcal{Z}$, such that the mutual dependence of two other statistical variables $\mathcal{W}$ and $\mathcal{D}$ is completely explained by their dependence from $\mathcal{Z}$. Formally:

$p\left(w_{i}, d_{j}\right)=\sum_{k} p\left(w_{i} \mid z_{k}\right) p\left(z_{k} \mid d_{j}\right) p\left(d_{j}\right)$

We now assume the following equalities:

$V_{i j} \approx c \cdot p\left(w_{i}, d_{j}\right)$

$W_{i k}=p\left(w_{i} \mid z_{k}\right)$

$H_{k j}=c \cdot p\left(z_{k} \mid d_{j}\right) \cdot p\left(d_{j}\right)$

with $c=\sum_{i j} V_{i j}$. Using these equations, it is clear that PLSA attempts to solve the same problem as NMF, since Eq. (4) is essentially the same as Eq. (1).

The parameters that need to be estimated in PLSA are $\theta=\left\{p\left(w_{i} \mid z_{k}\right), p\left(z_{k} \mid d_{j}\right)\right\}$. There is no need to estimate $p\left(d_{j}\right)$, as it can be determined by marginalizing $p\left(w_{i}, d_{j}\right)$. A solution for Eq. (4) can then be found based on the input data $\mathbf{V}$, according to a maximum likelihood criterion:

$\theta_{M L}=\underset{\theta}{\operatorname{argmax}} \log P(\mathbf{V} \mid \theta)$

This can be solved by applying the EM-algorithm. The expectation function of which is

$Q(\hat{\theta} \mid \theta)=\sum_{i=1}^{M} \sum_{j=1}^{N} V_{i j} \sum_{k=1}^{R} p\left(z_{k} \mid w_{i}, d_{j}\right) \log \left(\hat{p}\left(w_{i} \mid z_{k}\right) \cdot \hat{p}\left(z_{k} \mid d_{j}\right)\right)$

The maximization of this function with respect to $\hat{p}\left(w_{i} \mid z_{k}\right)$ and $\hat{p}\left(z_{k} \mid d_{j}\right)$ leads to the following update formulas:

$p\left(w_{i} \mid z_{k}\right) \leftarrow \frac{\sum_{j} V_{i j} p\left(z_{k} \mid w_{i}, d_{j}\right)}{\sum_{n} \sum_{j} V_{n j} p\left(z_{k} \mid w_{n}, d_{j}\right)}$

$p\left(z_{k} \mid d_{j}\right) \leftarrow \frac{\sum_{i} V_{i j} p\left(z_{k} \mid w_{i}, d_{j}\right)}{\sum_{l} \sum_{i} V_{i j} p\left(z_{l} \mid w_{i}, d_{j}\right)}$

in which after each update

$p\left(z_{k} \mid w_{i}, d_{j}\right)=\frac{p\left(w_{i} \mid z_{k}\right) \cdot p\left(z_{k} \mid d_{j}\right) p\left(d_{j}\right)}{\sum_{l} p\left(w_{i} \mid z_{l}\right) \cdot p\left(z_{l} \mid d_{j}\right) p\left(d_{j}\right)}$ 
Using Eqs. (6) and (7) it can be shown that the update algorithm in Eqs. (9a) and (9b) is equivalent to (see [25])

for I iterations do

$$
W_{i k} \leftarrow W_{i k} \cdot \sum_{j} H_{k j}\left(\frac{V}{W H}\right)_{i j}
$$

normalize : $\sum_{i} W_{i k}=1$

$H_{k j} \leftarrow H_{k j} \cdot \sum_{i} W_{i k}\left(\frac{V}{W H}\right)_{i j}$

normalize : $\sum_{k} H_{k j}=c \cdot p\left(d_{j}\right)=\sum_{i} V_{i j}$

The only difference with NMF is thus the normalization in Eq. (11d). However, this is hardly a conclusive distinction between the two algorithms, since it can also be applied in regular NMF. In fact, one can prove that when the normalization in (11b) is performed, (11d) minimizes the Kullback-Leiblerdivergence for the given values in $\mathbf{W}$ and $\mathbf{H}$. This means that normalizing $\mathbf{H}$ this way facilitates convergence, even in regular NMF. In conclusion, PLSA and NMF are based on two very different formulations that solve a similar problem (i.e. factorization). Whereas NMF uses KL-divergence as its cost function and minimizes it using multiplicative updates derived from gradient steps (see [9]), PLSA maximizes a likelihood using an EM-algorithm. It is remarkable that these algorithms lead to such similar iterative solutions.

\section{Adaptive PLSA}

Adaptivity is relevant to obtain cognitively plausible computational models. If we want to apply a NMF- or PLSA-based decomposition technique in a computational model of word learning, it is crucial that the data processing applied in such a model is compatible with the data processing as taking place in real-life learning. For reasons we will discuss below, in Section 7, we believe that a more realistic learning framework would process speech utterances one at a time, gradually updating and improving the word models based on recent data. Implementing such a framework requires an algorithm that can initialize and update models, without long term storage of speech input. To that end, we will here discuss a way to make NMF and PLSA adaptive, similar to the way it was proposed in [2]. Suppose that not all input data are available to the algorithm at once, but chunks of data $\mathbf{V}^{(\mathbf{n})}$ are presented in separate epochs, containing only a small number of columns. The exact number can vary, but in the experiments below we have used chunks of 1 column. It is then possible in each of these epochs to perform a Bayesian re-estimation of $\theta$, using the new input $\mathbf{V}^{(\mathbf{n})}$. The formal maximum likelihood criterion is

$\theta^{(n)}=\underset{\theta}{\operatorname{argmax}} \log \left(p\left(\mathbf{V}^{(\mathbf{n})} \mid \theta\right)\right)+\log \left(p\left(\theta \mid \mathcal{V}^{(n-1)}\right)\right)$

in which $\mathcal{V}^{(n)}$ is the set of $\left\{\mathbf{V}^{(\mathbf{1})}, \mathbf{V}^{(\mathbf{2})}, \ldots, \mathbf{V}^{(\mathbf{n})}\right\}$. The second term in this equation expresses the priors on $\theta$. It is a joint probability of the parameters to be estimated, which are assumed to be Dirichlet distributed. Naively, one could say that

$$
\begin{aligned}
p\left(\theta \mid \mathcal{V}^{(n-1)}\right)= & p\left(\theta_{W} \mid \mathcal{V}^{(n-1)}\right) \cdot p\left(\theta_{H} \mid \mathcal{V}^{(n-1)}\right) \\
& \propto \prod_{k=1}^{R}\left(\prod_{i=1}^{M} p\left(w_{i} \mid z_{k}\right)^{\alpha_{i k}-1} \cdot \prod_{j=1}^{M} p\left(z_{k} \mid d_{j}\right)^{\beta_{k j}-1}\right)
\end{aligned}
$$

in which $\alpha$ and $\beta$ are the hyperparameters of the respective distributions. However, we propose a number of changes to these equations. The columns of $\mathbf{H}$, which contain merely input-specific weights of word patterns, differ in every epoch. For our purposes, to establish acoustic patterns that relate to words, these columns contain no useful information. Hence, it makes no sense to transfer prior information about $\mathbf{H}$ into the current re-estimation of $\theta$. We therefore assume $p\left(\theta_{H} \mid \mathcal{V}^{(n-1)}\right)$ to be uniform, effectively turning it into a constant. Eq. (13) can then be rewritten as

$p\left(\theta \mid \mathcal{V}^{(n-1)}\right) \propto \prod_{k=1}^{R}\left(\prod_{i=1}^{M} p\left(w_{i} \mid z_{k}\right)^{\alpha_{i k}-1}\right)$

Furthermore, in order to avoid using all information from every single input ever seen in the current re-estimation of the models, we propose that the second term in Eq. (12), i.e. the prior distribution of $\theta$ should be scaled down with respect to the posterior probability of the current input. In practice, this means we can rewrite Eq. (12) as

$\theta^{(n)}=\operatorname{argmax} \log \left(p\left(\mathbf{V}^{(\mathbf{n})} \mid \theta\right)\right)+\gamma \log \left(p\left(\theta \mid \mathcal{V}^{(n-1)}\right)\right)$

in which $0 \ll \gamma<1$. Solving this maximum likelihood problem with the EM-algorithm then yields the new update formula:

$p\left(w_{i} \mid z_{k}\right) \leftarrow \frac{\sum_{j} V_{i j}^{(n)} p\left(z_{k} \mid w_{i}, d_{j}\right)+\gamma \kappa_{i k}^{(n-1)}}{\sum_{l} \sum_{j} V_{l j}^{(n)} p\left(z_{k} \mid w_{l}, d_{j}\right)+\gamma \kappa_{l k}^{(n-1)}}$

with $\kappa_{i k}=\alpha_{i k}-1$. The update formula for $p\left(z_{k} \mid d_{j}\right)$ remains equal to Eq. (9b). In every epoch the hyperparameters $\kappa$ need to be updated as well. It is shown in [2] that this can be done with the formula:

$\kappa_{i k}^{(n)}=\sum_{j} V_{i j}^{(n)} p\left(z_{k} \mid w_{i}, d_{j}\right)+\gamma\left(\kappa_{i k}^{(n-1)}\right)$

Note that due to the addition of the parameter $\gamma$ this value cannot increase without bounds. In summary, the algorithm for adaptive re-estimation of the word models becomes

for each epoch $n$ do

for I iterations do

$$
W_{i k} \leftarrow W_{i k} \cdot \sum_{j} H_{k j}\left(\frac{V^{(n)}}{W H}\right)_{i j}+\gamma \kappa_{i k}^{(n-1)}
$$

normalize : $\sum_{i} W_{i k}=1$

$H_{k j} \leftarrow H_{k j} \cdot \sum_{i} W_{i k}\left(\frac{V^{(n)}}{W H}\right)_{i j}$

normalize : $\sum_{k} H_{k j}=c \cdot p\left(d_{j}\right)=\sum_{i} V_{i j}^{(n)}$

$\kappa_{i k}^{(n)}=W_{i k} \sum_{j} H_{k j}\left(\frac{V^{(n)}}{W H}\right)_{i j}+\gamma \kappa_{i k}^{(n-1)}$

Notice the similarities between the updates of $\mathbf{W}$ and of $\kappa$. This means that $\kappa^{(n)}$ is the value of $\mathbf{W}$ that is obtained in epoch $n$ after convergence, the difference being that $\kappa$ is never normalized. In the very first epoch, $\kappa_{i k}^{(0)}$ is set equal to zero, since there is no prior information to use. In practice, this means that in the first epoch the adaptive algorithm is exactly the same as the classical batch algorithm, being run on $\mathbf{V}^{(\mathbf{1})}$. The initialization of $\mathbf{W}$ is more complicated and will be further described in Section 5.4.

\section{Complexity analysis}

When determining the computational load of these two algorithms, an important property to take into account is the sparsity 
Table 1

The total number of floating point operations for each individual update in batch NMF.

\begin{tabular}{llll}
\hline Eq. & \#divisions & \#other & Total \\
\hline$(11 \mathrm{a})$ & $s M N$ & $4 s M N R-s M N$ & $4 s M N R$ \\
$(11 \mathrm{~b})$ & $M R$ & $M R-R$ & $2 M R-R$ \\
$(11 \mathrm{c})$ & $S M N$ & $4 s M N R-s M N$ & $4 s M N R$ \\
$(11 \mathrm{~d})$ & $N$ & $2 N R-N$ & $2 N R$ \\
\hline
\end{tabular}

Table 2

The total number of floating point operations for each individual update in adaptive NMF.

\begin{tabular}{llll}
\hline Eq. & \#divisions & \#other & Total \\
\hline$(18 \mathrm{a})$ & $s M$ & $5 s M R+M R-s M$ & $5 s M R+M R$ \\
$(18 \mathrm{~b})$ & $M R-R$ & $M R$ & $2 M R-R$ \\
$(18 \mathrm{c})$ & $s M$ & $4 s M R-s M$ & $4 s M R$ \\
$(18 \mathrm{~d})$ & 1 & $2 R-1$ & $2 R$ \\
$(18 \mathrm{e})$ & $s M$ & $5 s M R+M R-s M$ & $5 s M R+M R$ \\
\hline
\end{tabular}

of the input data. We define the sparsity parameter $s$ as

$$
s=\frac{\# \text { non }- \text { zero elements of } \mathbf{V}}{M \cdot N}
$$

Typical values of $s$ in our experiments were around 0.01. In the following calculations, we will make the assumption for row operations on $\mathbf{V}$ that each row contains $s N$ non-zero values. Likewise, for column operations on $\mathbf{V}$ we assume there are $s M$ non-zero values in each column of $\mathbf{V}$. Operations involving zeros that do not affect the outcome of the calculations are not counted. As an example, in the matrix multiplication $(\mathbf{V} / \mathbf{W H}) \cdot\left(\mathbf{H}^{T}\right)$ we count $M R$ times the inner product of two vectors with length $s N$, yielding a total of $s M N R$ multiplications and $M R(s N-1)$ additions, or $2 s M N R-M R$ floating point operations. The number of operations for the different update formulas of both algorithms are shown in Tables 1 and 2. Because on most platform divisions are more expensive than other floating point operations, the number of divisions in each update step is also shown. For the adaptive algorithm, we have assumed that in each epoch, $\mathbf{V}$ contains only a single column.

We can conclude that, if $I$ is the number of iterations, the batch algorithm requires $I \cdot(8 s M N R+2 M R+2 N R-R)$ floating point operations. The adaptive algorithm, where $I$ is the number of iterations per epoch, requires $N \cdot(I \cdot(9 s M R+3 M R+R)+5 s M R+M R)$ operations. It appears that the adaptive NMF-algorithm is slower since it has a complexity of $O(M N R I)$ as opposed to the batch algorithm which has a complexity of $O(S M N R I)$. The difference originates from the normalization of the columns in $\mathbf{W}$. This operation has to be performed $\mathrm{NI}$ times in the adaptive algorithm and only I times in the batch algorithm. In practice, however, the difference in complexity is lower than it may seem, since the parameter $I$ in adaptive NMF is typically chosen much lower than in batch NMF.

\section{A computational model for vocabulary acquisition}

\subsection{The structure of $\mathbf{V}$}

The speech data on which we will apply this learning algorithm will consist of a large set of speech utterances, each of which will contain a keyword that is to be added to the learned vocabulary. In order for these keywords to be learned, a data matrix $\mathbf{V}$ has to be constructed based on this speech data. In practice, this means we will start by converting each of the speech utterances into a single vector of fixed size. In our experiments, this is done by using the HAC-representation (see below).

In order for the computational framework to attach meaning to speech patterns discovered in these utterances, information about the scene from other modalities than speech, i.e. grounding information, has to be added to them. This complicates the task of learning, since processing all this information is an extremely difficult problem in its own right. For instance, having a computational algorithm reliably detect patterns from visual images is a problem at least as complex as the detection of words from speech. Since we wish to limit the scope of this paper to the processing of acoustic information, we will make abstraction of this difficulty and simplify the representation of the multi-modal data: we will assume that all information from other modalities than speech is somehow perfectly recognized, always present when spoken about in an utterance and always the only thing that is present. Concretely, this means that we will attach a socalled multi-modal tag to each utterance, indicating the keyword contained in that utterance. For instance, when the speech data contains the sentence "Mummy is driving", we will attach the tag $\langle$ mummy > to it.

All information pertaining to a single utterance can be bundled in a single vector if the presence of a multi-modal tag with an utterance is vectorized as well. The vectors constructed in this way from all available speech utterances are then combined as the columns of a single data matrix $\mathbf{V}$, which will be structured in our experiments as

$\mathbf{V}=\left[\frac{\mathbf{V}_{\mathbf{g}}}{\mathbf{V}_{\mathbf{H A C}}}\right]$

in which $\mathbf{V}_{\mathbf{g}}$ is the grounding part and $\mathbf{V}_{\mathbf{H A C}}$ the acoustic part.

\subsection{Acoustic information: the HAC-representation}

NMF requires the representation of a speech utterance that is

- a vector of fixed length;

- non-negative;

- additive, i.e. the representation of an utterance should be a (weighted) summation of the representations of its constituent parts.

The Histogram of Acoustic Co-occurrences (HAC), proposed in [27] is such a representation. In Fig. 4 the process of converting a single speech utterance into the HAC-representation is schematically shown. Twenty ms frames with a frame shift of $10 \mathrm{~ms}$ are taken from the signal. In each frame 12 MFCCs (mel-frequency cepstral coefficients, see [20]) are calculated. These parameters form a compact representation of the spectral information within that frame, according to an auditory model. Along with the energy, there are thus 13 parameters that describe the static spectral shape of the signal within each frame. First and second order differences of these values can be taken with respect to time to derive spectral velocities $(\Delta)$ and accelerations $(\Delta \Delta)$ of the speech signal. Vector quantization is performed on these data streams, in order to convert them into sequences of labels. The size of the necessary vector codebooks can be freely chosen. Sizes between 100 and 200 yield good trade-off between complexity and accuracy in modelling the speech signal. The codebooks are trained by performing kmeans-clustering on the frames of all utterances in the database, including both train set and test set. Having converted the speech signal into three separate label sequences, co-occurrences of these labels at a time offset $\tau$ can be counted and put into a histogram. This time offset, which must be a multiple of the frame shift, determines the distance between two labels at which their co-occurrences are counted. 


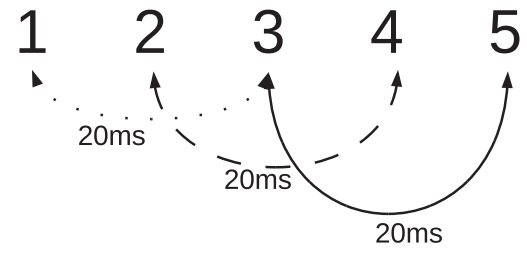

Fig. 3. Co-occurrences at a time offset of $20 \mathrm{~ms}$ in a label sequence where every label describes $10 \mathrm{~ms}$ of the speech signal.

For instance, if we have a label sequence $(1,2,3,4,5)$, a frame shift of $10 \mathrm{~ms}$ and $\tau=20 \mathrm{~ms}$, the co-occurrences that are put into the histogram would be: $1|3,2| 4$ and $3 \mid 5$, see Fig. 3 . For a codebook with cardinality $L$, this histogram will have a length $L^{2}$. Multiple time offsets can be considered on each of the three data streams: statics, velocities and accelerations. The concatenation of all the resulting vectors is then used as one column in $\mathbf{V}_{\mathbf{H A C}}$.

\subsection{Grounding information}

Every column of the matrix $\mathbf{V}_{\mathbf{g}}$ belongs with an utterance and therefore with a column in $\mathbf{V}_{\text {HAC. }}$. Each column in $\mathbf{V}_{\mathbf{g}}$ has a length equal to the number of keywords that exist within the data, each position in the vector corresponding to one of them. The element at the position that corresponds with the keyword in its designated utterance is 1 . For the rest, each column is filled with 0's. Formally,

$V_{g}(i, j)= \begin{cases}1 & \text { if utt. } j \text { contains keyword } i \\ 0 & \text { otherwise }\end{cases}$

\subsection{Training word models}

\subsubsection{Batch training}

Training models for word-like patterns then becomes a matter of factorizing the data matrix $\mathbf{V}$ into matrices $\mathbf{W}$ and $\mathbf{H}$ with a well-chosen inner dimension. Since it is our goal to have each keyword modelled by at least one column in $\mathbf{W}$, this inner dimension should be at least equal to the number of possible keywords. Extra columns in $\mathbf{W}$ are necessary, however, to accommodate co-occurrences in the data that are unrelated to any of the keywords (carrier sentence, silence between words, cross-word co-occurrences, etc.), further also referred to as garbage. We will make a distinction between the rows in $\mathbf{W}$ that correspond to rows in $\mathbf{V}_{\mathbf{g}}$, containing semantic information, and those that correspond to rows in $\mathbf{V}_{\mathbf{H A C}}$, containing acoustic information. We can rewrite Eq. (1) as follows:

$\left[\begin{array}{c|c}\mathrm{V}_{\mathrm{g}} \\ \hline \mathrm{V}_{\mathrm{HAC}}\end{array}\right] \approx\left[\begin{array}{c|c}\mathrm{W}_{\mathrm{g}} & \mathrm{G}_{\mathrm{g}} \\ \hline \mathrm{W}_{\mathrm{HAC}} & \mathrm{G}_{\mathrm{HAC}}\end{array}\right] \cdot\left[\begin{array}{c}\mathrm{H}_{\mathrm{w}} \\ \hline \mathrm{G}_{\mathrm{H}}\end{array}\right]$

NMF is only proven to find a local optimum of its cost function, not a global one (see $[9,21]$ ). As such, the initialization of these matrices is important, since it influences the local optimum NMF converges towards. Each of the submatrices of $\mathbf{W}$ and $\mathbf{H}$ have a different role to play in the algorithm:

- $\mathbf{W}_{\mathbf{g}}$ : The grounding part of the columns that pertain to words. Each column consists of very small random values with a single large one $(\approx 1)$ indicating the keyword of which the acoustic representation is present in the corresponding column of $\mathbf{W}_{\text {HAC. }}$ In practice, this means that $\mathbf{W}_{\mathbf{g}}$ will be an identity matrix, with small positive random noise added to it.

- $\mathbf{G}_{\mathbf{g}}$ : The grounding part of the columns that pertain to garbage. It is initialized containing small positive random values.

- $\mathbf{H}_{\mathbf{w}}$ : This matrix contains in its columns the weights of the keywords in the utterances of the input. At initialization time it is set equal to $\mathbf{V}_{\mathbf{g}}$. It is updated during the iterations of NMF, but will never digress far from its initial value, since that is the optimum for

$\mathbf{V}_{\mathbf{g}} \approx \mathbf{W}_{\mathbf{g}} \mathbf{H}_{\mathbf{w}}$

- $\mathbf{W}_{\text {HAC: }}$ The acoustic part of the columns that pertain to keywords. It is initialized with comparatively large random values. It will in the end contain HAC-representations of the keywords, due to the initializations of $\mathbf{W}_{\mathbf{g}}$ and $\mathbf{H}_{\mathbf{w}}$, which provide supervision.

- $\mathbf{G}_{\mathbf{H A C}}$ and $\mathbf{G}_{\mathbf{H}}$ : These are randomly initialized. They will converge towards values that optimally approximate the sum of all co-occurrences that are not keyword-related:

$\mathbf{G}_{\mathrm{HAC}} \cdot \mathbf{G}_{\mathbf{H}} \approx \mathbf{V}_{\mathrm{HAC}}-\mathbf{W}_{\mathrm{HAC}} \cdot \mathbf{H}_{\mathrm{w}}$

It is clear that at the end of the factorization, $\mathbf{W}$ is what we will be interested in, since it contains general representations of the keywords and of the garbage that surrounds them.

\subsubsection{Adaptive training}

When working in an adaptive way, $\mathbf{V}^{(\mathbf{n})}$ and $\mathbf{H}^{(\mathbf{n})}$ in each epoch $n$, will be slices of $\mathbf{V}$ and $\mathbf{H}$ containing only a small number of columns (in our experiments typically only one). The initialization of $\mathbf{V}$ and $\mathbf{H}$, as well as the matrix $\mathbf{W}$, is done in the same way as for the batch algorithm, explained in Section 5.4.1. After processing data for enough epochs, $\mathbf{W}$ will converge towards a value similar to the one attained with batch processing.

\subsection{Prediction}

The trained $\mathbf{W}$-matrix, which we will refer to as $\mathbf{W}^{\text {(trained) }}$ for the rest of this section, can be used to identify which keyword is present in unseen test utterances. If the HAC-representations of these utterances form the data matrix $\mathbf{V}_{\mathbf{H A C}}^{\text {(test) }}$, NMF can be run in a one-sided way, iteratively updating a randomly initialized H-matrix while leaving $\mathbf{W}^{\text {(trained) }}$ constant, such that

$\mathbf{V}_{\mathbf{H A C}}^{(\text {test })} \approx\left[\mathbf{W}_{\mathrm{HAC}}^{(\text {trained) }} \mid \mathbf{G}_{\mathrm{HAC}}^{\text {(trained) }}\right] \cdot\left[\frac{\mathbf{H}_{\mathrm{w}}^{\text {(test) }}}{\mathbf{G}_{\mathbf{H}}^{\text {(test) }}}\right]$

Mind that, since this one-sided algorithm has a convex cost function, the resulting $\mathbf{H}$-matrix approaches a unique solution.

It is then finally possible to calculate an activation matrix

$$
\mathbf{A}=\mathbf{W}_{\mathrm{g}}^{\text {(trained) }} \cdot \mathbf{H}_{\mathrm{w}}^{\text {(test) }}
$$

$A$ can be seen as a prediction of what $\mathbf{V}_{\mathbf{g}}^{(\text {test })}$ should look like given the trained models in $\mathbf{W}_{\mathbf{H A C}}^{\text {(trained) }}$. As such, the selected keyword in a given utterance of the test set is the one indicated by the highest value in the corresponding column of $A$. Since there is only one keyword to be detected in each utterance the only errors that the algorithm can make are substitution errors. Over the entire test set, a keyword error rate (KER) can be calculated to quantify the accuracy of the trained models in $\mathbf{W}_{\text {HAC }}^{\text {(trained) }}$.

\section{Experiments}

For the experiments, we made use of a database that was recorded for the ACORNS-project (see [29]). This project aimed at investigating a number of computational models of language acquisition, NMF among them, so this data is very suitable for the purpose we wish to use it for. It consists of 4000 British English spoken utterances, two male and two female speakers having contributed 1000 utterances each. Of the 1000 utterances provided by each speaker, half is spoken as if directed to a child (infantdirected speech), the other half is spoken normally (adult-directed 
speech). This is meant to model the kind of speech data a real infant is confronted with at a very young age. The utterances have a very simple syntactic structure, containing a single keyword enveloped by a carrier sentence. The keywords in this database are selected from a limited set: "Angus", "Ewan”, "bath", "book", "bottle”, "car", "daddy", "mummy", "nappy", "shoe" and "telephone". An example of a typical utterance in the database is: "Mummy is driving". Three thousand utterances are selected at random and make up the train set, the remaining 1000 make up the test set.

These utterances are then all converted into HAC-vectors. To this end, the MFCCs and their first and second order differences are converted into three sequences of labels: statics, velocities and accelerations. The vector codebooks used in this operation are of size 150 for the statics, 150 for the velocities and 100 for the accelerations. Co-occurrences within these label sequences are counted at three different time offsets: $\tau_{1}=20 \mathrm{~ms}, \tau_{2}=50 \mathrm{~ms}$ and $\tau_{3}=90 \mathrm{~ms}$. Concatenating the resulting histograms for each stream yields for every utterance a vector of length $3 \times 150^{2}$ with static information, $3 \times 150^{2}$ for velocity information and $3 \times 100^{2}$ for the acceleration information. Bundling the HAC-vectors of the train and test set leads to the resulting matrices $\mathbf{V}_{\text {HAC }}^{\text {(train) }}$ and $\mathbf{V}_{\mathrm{HAC}}^{\text {(test) }}$ with respective dimensions $165000 \times 3000$ and $165000 \times 1000$. Since there are 11 keywords to be detected, the grounding matrix $\mathbf{V}_{\mathbf{g}}$ that is added to $\mathbf{V}_{\mathrm{HAC}}^{(\text {train) }}$ has size $11 \times 3000$.

In our experiments, the inner dimension of the factorization was set to 20 . We have assigned nine columns to model garbage. $\mathbf{W}_{\mathbf{g}}$ is set at initialization as an $11 \times 11$ identity matrix, with very small random noise added $(O(1 e-4))$. $\mathbf{G}_{\mathrm{g}}$ was filled with random values of approximately the same size. $\mathbf{W}_{\mathbf{H A C}}$ and $\mathbf{G}_{\mathrm{HAC}}$ were filled with larger random values $(O(1))$ and offset with a value of 0.02 to avoid 0's. Those cannot be altered by multiplicative updates, which would reduce the flexibility of the models. $\mathbf{G}_{\mathbf{H}}$ was also randomly initialized. For the adaptive training, the matrices $\mathbf{V}$ and $\mathbf{H}$, from which a slice of only 1 column is used in every epoch, were initialized with the exact same random values as in batch training, avoiding any initialization-related influence on the results from the two algorithms, which would make any comparison less meaningful.

Finally, the testing of the trained models is performed as described in Section 5.5. A suitable matrix $\mathbf{H}^{\text {(test) }}$ is created by randomly initializing a matrix of size $20 \times 1000$ and updating it 30 times. With this matrix, the activation matrix $A$ with size $11 \times 1000$ can easily be calculated using Eq. (20).

\subsection{Experiment 1: learning rate}

The most important characteristic of the adaptive learning algorithm, when compared to the batch algorithm, is the way input data is used to form models. Information from each column is introduced into the models and gradually attenuated as more columns are processed. The question is how efficiently this method makes use of the available information. Will it produce models that are as accurate as those from the batch algorithm using the same amount of data?

In this experiment, adaptive PLSA is performed on the 3000 utterances of the train set. The number of iterative updates per epoch, $I$, is set equal to 10 . Thus, in every epoch, 10 iterative updates are performed using Eqs. (18a)-(18d), for $\gamma=1,0.9,0.99$ and 0.999 . Every 20 epochs, the accuracy of the models is measured by determining the KER on the test set, using the method described in Section 5.5. For comparison, the batch algorithm is run in the same epochs, according to Eqs. (11a)-(11d). The number of iterations, $I$, for these batch calculations is set equal to 700 . The resulting batch models were evaluated on the same test set as the adaptive models. All results are shown in Fig. 5.

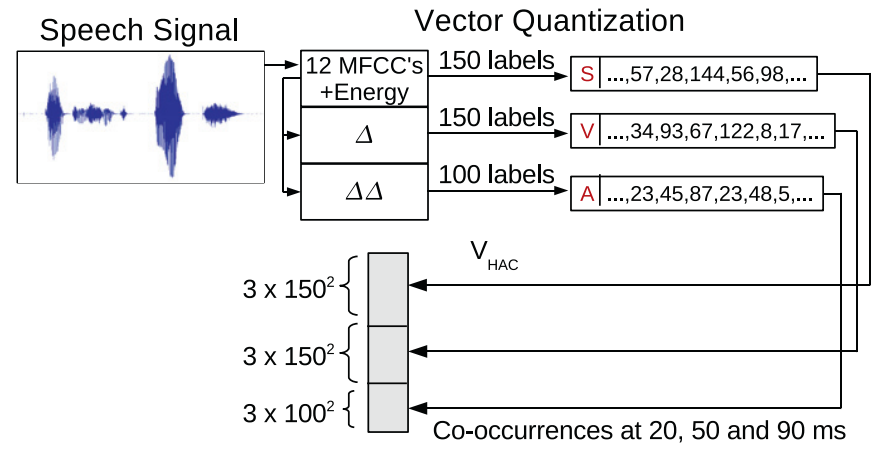

Fig. 4. Schematic overview of the conversion of a single speech utterance into a HAC-vector.

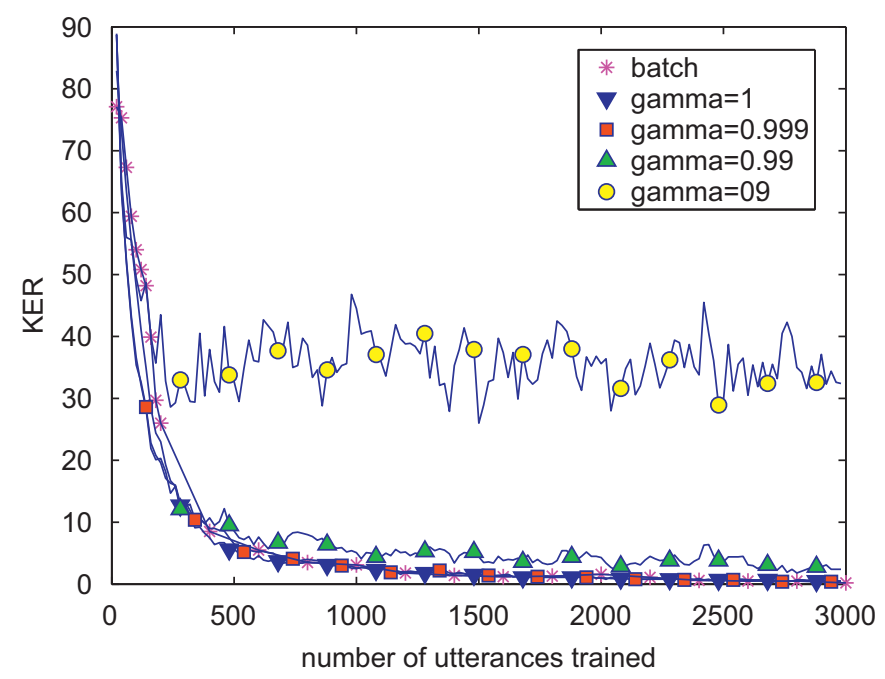

Fig. 5. Keyword error rates achieved on a set of unseen utterances, with increasing amounts of training data trained upon by the learning framework (both batch and adaptive).

The value of $\gamma$ turns out to be very influential on the end result. This is not so surprising since it indirectly determines the size of the memory in the adaptive learning algorithm, i.e. the number of past utterances that significantly influence the current models. Any value $x$ added to the models $m$ epochs ago will be reduced at present to a value $\gamma^{m} \cdot x$. Making use of a geometric series, it is then clear that this virtual memory length will be proportional to $1 /(1-\gamma)$. For low values of $\gamma$, this number is low, leading to models that are less accurate when compared to those obtained with high values of $\gamma$. Interesting to note is that for high values of $\gamma$, the adaptive algorithm yields results that are comparable to those obtained with the batch algorithm using the same amount of data, eventually settling on a KER of approximately $0.3 \%$. Intuitively, one might expect adaptively learned models to be suboptimal but this is not the case.

\subsection{Experiment 2: adaptation}

An important feature of the proposed learning framework is its ability to adapt to external changes. If the acoustic properties of a word in the acquired vocabulary change, the accuracy with which that word can be detected will decrease. The adaptive algorithm has to be able to correct the model it has learned for that word and has to do so automatically, without any external interference. In this experiment, we have rerun the experiment from Section 6.1 for $\gamma=1,0.9,0.99$ and 0.999 . After training for 3000 epochs, the semantics of the word representations in $\mathbf{W}$ were altered in 


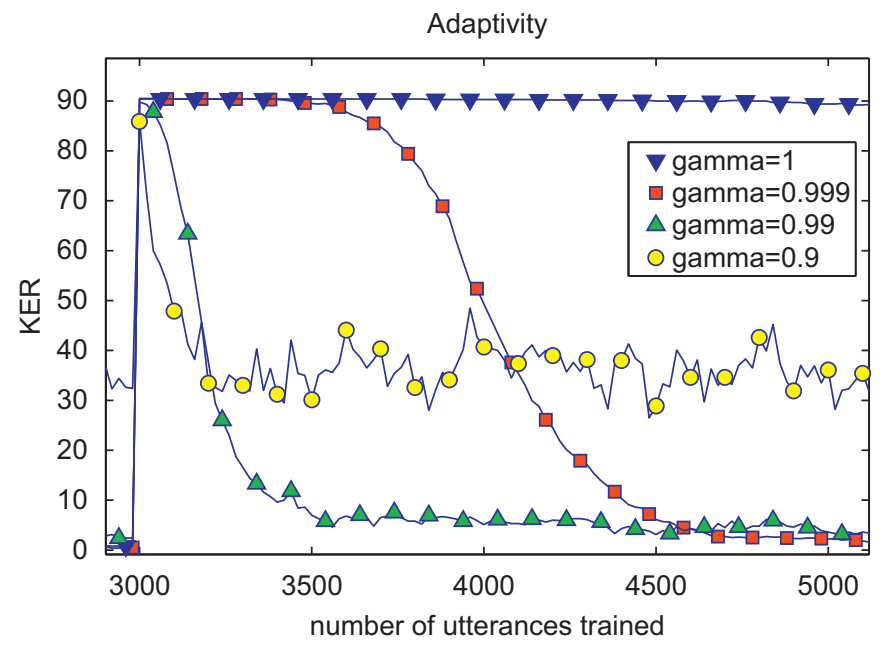

Fig. 6. Recovery of the keyword error rates achieved on a set of unseen utterances, after the semantics in the input data changed drastically.

such a way that everything the algorithm has learned up to that point is wrong. For instance, the acoustic representation of the word "shoe" may suddenly be linked to the tag $\langle$ mummy $\rangle$, "Ewan" to 〈bottle >, etc. Algorithmically, this is done by rearranging the rows in $\mathbf{W}_{\mathbf{g}}$. The learning is then continued by once more presenting the 3000 utterances of the training set to the algorithm. The KER on the test set is still determined by every 20 epochs. The result can be seen in Fig. 6 .

As expected, changing the meaning of the learned patterns makes the KER rise to the level of chance (90.91\%). The memory length, determined by the parameter $\gamma$, plays a key role in the way the algorithm recovers from this. The higher its value, the longer it takes to adapt the learned models. Interesting to note is the situation where $\gamma=1$. Adaptation to external changes in that situation turns out to be much slower. Since in that situation all inputs ever seen are equally important, the number of epochs required to unlearn word representations will be approximately the same as the number of epochs that were spent learning them, because only then the new meanings will swamp the old meanings. Although in real life learning situation changes will seldom be as profound as in this experiment, this does illustrate how important it is to take the recency of perceived inputs into account.

\subsection{Experiment 3: emergence of relevant acoustic patterns}

In the previous experiments we showed that the acquisition of new information and the attenuation of old information in the models give rise to a constant dynamic of learning and adaptation within the framework, the extent of which is regulated by the $\gamma$-parameter. The contents of the word models themselves have so far not been considered. The question we ask ourselves is how these models evolve from completely random vectors towards statistically meaningful word representations, while learning takes place. We conjecture that in the initial learning phase, features that belong to carrier sentences are mixed into the models of the words. As learning continues, these features are gradually effaced from the word models, leaving only keywordrelated patterns. In this experiment, adaptive training is again performed on the 3000 utterances of the train set, with $\gamma=0.999$, starting from randomly initialized word models. The first utterance, containing the sentence "Mummy is driving", is split into two parts: one containing the keyword "Mummy", the other containing the carrier sentence "is driving". These two acoustic signals are then converted into HAC-vectors $V_{\text {" }}^{(\text {test })}$ mumm” and $V_{\text {“ }}^{(\text {test })}$ is_driving". In every epoch the activation of the keyword $\langle$ mummy $\rangle$ by these acoustic signals is calculated similarly to how it was done in Section 5.5. For instance, the activation brought about by $V_{\text {“ }}^{(\text {(test })}$ is_driving" is calculated as follows: the column vector $H_{w, " ~}^{(t e s s}$ is_driving" is determined as part of the solution for

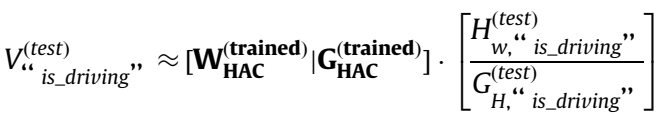

The activation is then

a“" is_driving" $=W_{g,\langle\text { mummy }\rangle} \cdot H_{w, " \text { is_driving" }}^{(\text {test })}$

in which $W_{g,\langle\text { mummy }\rangle}$ is the row in $\mathbf{W}_{\mathbf{g}}$ that corresponds with the keyword 〈mummy $\rangle$. The activation brought about by $V_{\text {“ }}^{(t e s t)}$ mummy" is calculated in a similar way.

The results for the first 500 epochs are shown in Fig. 7. They show that the learning framework's initial model for the keyword includes features from the keyword itself and features from the carrier sentence, since the acoustics of both the keyword and the carrier sentence activate the keyword equally. Eventually, the algorithm automatically starts to shift the focus of the word model towards the acoustic features of the word itself. This happens fairly quickly, after only 30 epochs. From that point onwards, the acoustics of the carrier sentence "is driving", no longer activate the acquired model for $\langle$ mummy $>$ to such a great extent. The activation of the keyword by the acoustics of its first pronunciation eventually also settles on a lower level than in the beginning. This makes sense, since the word model is generalized over multiple pronunciations of the keyword by multiple speakers.

One might argue that there is a trivial reason why this behaviour is observed. Specifically, the fact that all utterances containing the keyword 〈mummy〉, all with different carrier sentences, are mixed together, leading the ever changing garbage to be averaged out of the models. To determine how important this effect is in these results, we have set up another experiment in which keyword models, i.e. the columns of $\mathbf{W}_{\text {HAC, }}$, were created by accumulating input vectors. Concretely, if in epoch $n$ an utterance containing for instance the keyword 〈shoe〉 is observed, the following update is performed:

$W_{H A C,\langle\text { shoe }\rangle}=\gamma \cdot W_{H A C,\langle\text { shoe }\rangle}+\mathbf{V}_{\mathbf{H A C}}^{(\mathbf{n})}$

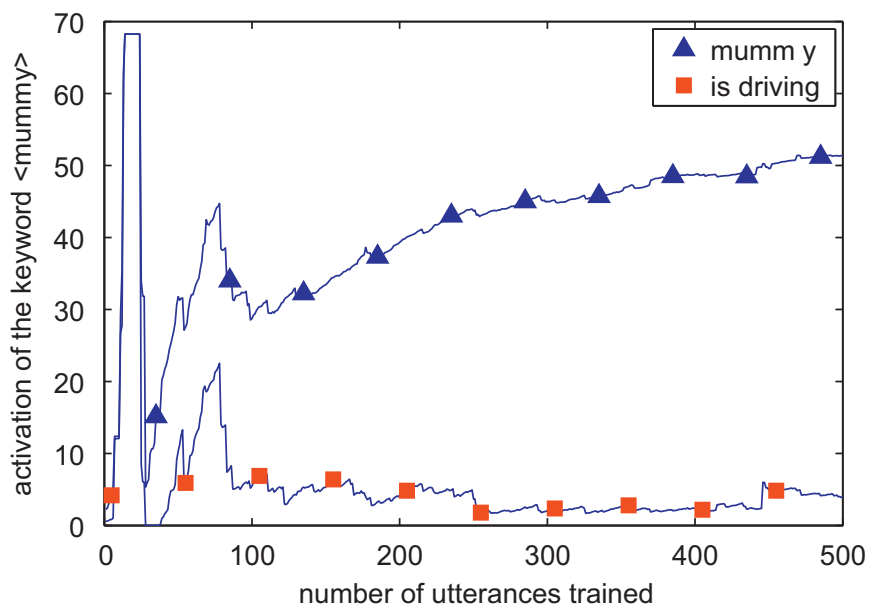

Fig. 7. Evolution of the activations of the keyword $\langle$ mummy $\rangle$, triggered respectively by the acoustics of the word "mummy", and by the acoustics of the carrier sentence "is driving". 


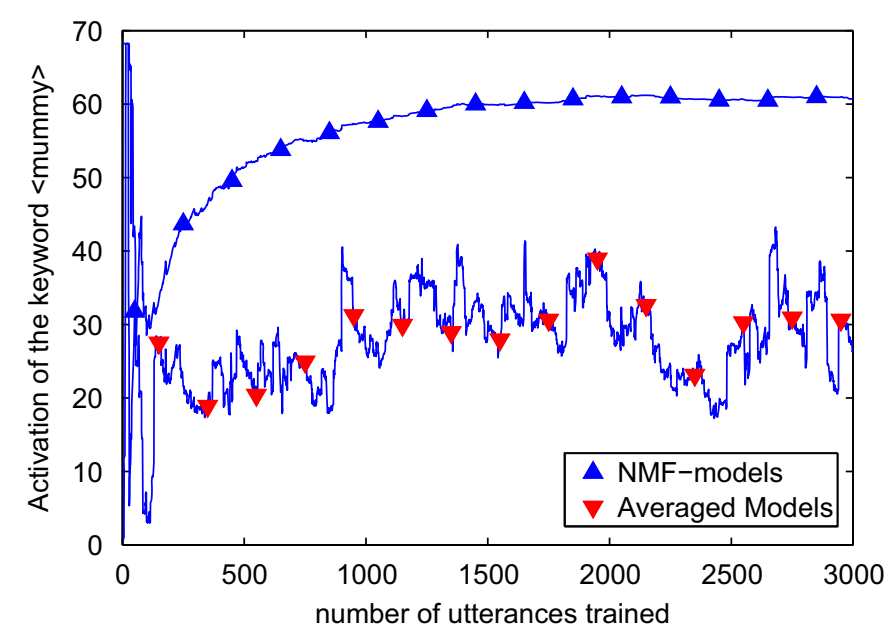

Fig. 8. Evolution of the activations of the models for keyword $\langle$ mummy $\rangle$, triggered by the acoustics of the word "mummy". One model is obtained by adaptive PLSA, the other is obtained by the accumulation of input vectors containing $\langle$ mummy $\rangle$.

in which $W_{H A C,\langle\text { shoe }}$ is the column of $\mathbf{W}_{\text {HAC }}$ that models the keyword $\langle$ shoe $\rangle$ and $\gamma=0.999$. The activation of the model for $\langle$ mummy $\rangle$, given the same acoustic pronunciation "mummy" as in the first experiment was then calculated in each epoch in the same way as before. In Fig. 8, the results for 3000 epochs are shown along with the activations of the NMF model for keyword $\langle$ mummy $\rangle$. These results show that merely averaging over all input utterances containing the correct keyword cannot explain the success of the models obtained with the adaptive framework.

\section{Discussion}

Detecting word-like patterns within speech signals augmented with information from other modalities has been shown to be possible with a NMF-based computational learning framework [27,28]. However, NMF is a batch algorithm, requiring a large amount of data to be processed at once. Batch NMF, and by extension any other batch learning algorithm that attempts to model vocabulary acquisition in infants with a claim at cognitive plausibility, can only do so by accepting a number of assumptions:

- Infants subconsciously store the speech stimuli into their long term memory with sufficient detail to facilitate learning, along with episodic information about their surrounding environment at that moment.

- The details of this stored information do not fade or change over time, i.e. they are not forgotten.

- All speech inputs, regardless of how long ago they were received, are equally important in the training of word models.

The adaptive learning framework proposed in this paper is capable of acquiring a basic vocabulary, while requiring none of these assumptions. Moreover, it can be more readily related to certain theories and models concerning memory and learning.

For instance, the structure of the long term memory in the batch framework seems overly simplistic. It essentially models the role of long term memory in vocabulary acquisition to perform two separate functions: the first is to store very detailed episodic traces of speech input and extra information about the environment (i.e. the matrix $\mathbf{V}$ in the learning framework), the second is to store the models used for the recognition of keywords (i.e. the matrix $\mathbf{W}$ in the learning framework). The first can be related to episodic memory in real infants, the second with semantic memory. However, the claim was made that these two kinds of memory cannot be viewed so cleanly separate from each other (e.g. [15,16]). The adaptive framework provides an alternative approach. In the experiment of Section 6.3, we have shown an example where the model for the keyword 〈mummy is initially no more than the episodic storage of the first utterance with which that keyword co-occurred, including silence, carrier sentence, intonation, etc. With training, this information was modified into a general acoustic word model with which the presence of the keyword can reliably be detected in utterances unseen during training. As such, it is shown that what could be seen within the framework as episodic memories, as they are mixed, linked and combined with each other, give rise to knowledge that can be seen as semantic. This also corresponds to the observation that, despite the fact that they have more limited processing and memory capabilities than adults [17], children learning speech initially tend to create very extensive and detailed representations of everything they hear, including information that might be irrelevant. Only later do they relax these representations into more general word models [3].

Another conceptual shortcoming of the batch framework is the fact that it disregards the importance of short term memory. There is ample evidence that the short term storage of phonological information plays an important role in vocabulary acquisition of real infants [10-14]. This is addressed in the adaptive framework, where detailed phonological and situational information, represented by the multi-modal tag, are buffered for a short period while being processed, after which they are discarded. This mechanism is especially consistent with Baddeley's theory of working memory [10-12].

Another strength of the adaptive framework is that it provides a simple model for forgetting. Due to the downscaling of the prior term in Eq. (14), all information within the long term memory of the learning framework decays exponentially when more inputs are processed. This principle of weakening old memories when new ones are stored relates roughly with the interference model of forgetting $[18,19]$. It was shown in the experiment of Section 6.2 that this property of the framework was crucial for the rapid adaptation of its acquired knowledge to structural changes in the input.

We realize that many of the cognitive and psychological theories mentioned here, are still the topic of ongoing debate and controversy in the field of psychology. We stress that it is by no means our intention to make claims about the validity of any of them. However, the main purpose of introducing adaptive learning into this NMF-based model of vocabulary acquisition is to improve its cognitive plausibility. If in that regard the adaptive framework is to be compared with the batch one, it is important that it can be convincingly positioned more closely to views about memory and learning in cognitive theory. We have argued that this is the case, even if these views are not shared by every single expert in the field.

\section{Conclusion}

In this paper, we have enabled a NMF-based computational framework for vocabulary acquisition to learn adaptively. To this end, the link between NMF and PLSA was thoroughly explored. Since the concept of Bayesian inference can readily be applied to PLSA, fully understanding the similarity between these two algorithms enabled us to extend these principles to NMF. The adaptive algorithm was experimentally compared to the batch algorithm in terms of accuracy, showing that adaptivity can be introduced without losing performance. Furthermore, we showed 
that the adaptive framework is capable of adapting automatically to even radical changes in the input and investigated how the word models evolve as learning is performed. Finally, we discussed the fact that the proposed adaptive framework is cognitively more plausible than its batch counterpart, since it depends on fewer assumptions. Furthermore, we used the experimental results to illustrate that it is far more consistent with existing models and theories in psychology concerning memory and learning.

\section{Future work}

The experiments performed with the proposed learning framework in this paper form a basis to establish its conceptual advantages. Future work may include such things as

- The acquisition of larger vocabularies.

- More realistic representations of information from other modalities than speech.

- More syntactically challenging utterances (e.g. no fixed number of keywords in each utterance).

- More elaborate models for forgetting.

\section{Acknowledgements}

This research was funded by the European commission (ACORNS project, FP6-034362) and the Research Fund K.U.Leuven (VASI project, OT/09/028).

\section{References}

[1] P.O. Hoyer, Non-negative matrix factorization with sparseness constraints, Journal of Machine Learning Research 5 (2004) 1457-1469.

[2] J.-T. Chien, M.-S. Wu, Adaptive Bayesian latent semantic analysis, IEEE Transactions on Audio, Speech and Language Processing 16 (2008) 198-207.

[3] R. Newman, The level of detail in infant's word learning, Current Directions in Psychological Science 17 (3) (2008) 229-232.

[4] P.K. Kuhl, Early language acquisition: cracking the speech code, Nature Reviews Neuroscience 5 (2004) 831-843.

[5] J.R. Saffran, D.P. Wilson, From syllables to syntax: multilevel statistical learning by 12 -month-old infants, Infancy 4 (2003) 273-284.

[6] P.W. Jusczyk, How infants begin to extract words from speech, Trends in Cognitive Sciences 3 (1999) 323-328.

[7] J.F. Werker, H.H. Yeung, Infant speech perception bootstraps word learning, Trends in Cognitive Science 9 (11) (2005) 519-527.

[8] D.D. Lee, H.S. Seung, Learning the parts of objects by non-negative matrix factorization, Nature 401 (1999) 788-791.

[9] D.D. Lee, H.S. Seung, Algorithms for non-negative matrix factorization, Advances in Neural Information Processing 13 (2001).

[10] A.D. Baddeley, G.J.L. Hitch, Working memory, in: G.H. Bower (Ed.), The Psychology of Learning and Motivation: Advances in Research and Theory, vol. 8, 1974

[11] A. Baddeley, Working memory model: looking back and looking forward, Nature Reviews Neuroscience 4 (10) (2003) 829-839.

[12] A. Baddeley, S. Gathercole, C. Papagno, The phonological loop as a language learning device, Psychological Review 105 (1) (1998) 158-173.

[13] S.E. Gathercole, A. Baddeley, Evaluation of the role of phonological STM in the development of vocabulary in children: a longitudinal study, Journal of Memory and Language 28 (2) (1989) 200-213.

[14] E. Service, V. Kohonen, Is the relation between phonological memory and foreign language learning accounted for by vocabulary acquisition?Applied Psycholinguistics 16 (1995) 155-172
[15] G. McKoon, R. Ratcliff, G.S. Dell, A critical evaluation of the semantic-episodic distinction, Journal of Experimental Psychology: Learning, Memory, and Cognition $16(2)$

[16] L. Ryan, C. Cox, S.M. Hayes, L. Nadel, Hippocampal activation during episodic and semantic memory retrieval: comparing category production and category cued recall, Neuropsychologia 46 (8) (2008) 2109-2121.

[17] C.L. Stager, J.F. Werker, Infants listen for more phonetic detail in speech perception than in word-learning tasks, Nature 388 (1997) 381-382.

[18] J.B. Jenkins, K.M. Dallenbach, Oblivescence during sleep and waking, American Journal of Psychology 35 (1924) 605-612.

[19] J.A. McGeoch, Forgetting and the law of disuse, Psychological Review 39 (1932) 352-370.

[20] S.B. Davis, P. Mermelstein, Comparison of parametric representations for monosyllabic word recognition in continuously spoken sentences, IEEE Transactions on Audio, Speech and Signal Processing 28 (1980) 357-366.

[21] Hans Laurberg, Mads Græsbøll Christensen, Mark D. Plumbley, Lars Ka Hansen, and Søren Holdt Jensen, Theorems on positive data: on the uniqueness of NMF, Computational Intelligence and Neuroscience, 2008 (2008) 9 doi:10.1155/2008/764206.

[22] D. Roy, A. Pentland, Learning words from natural audio-visual input, in: Proceedings of the International Conference of Spoken Language Processing, 1998.

[23] L. Ten Bosch, H. Van hamme, L. Boves, A computational model of language acquisition: focus on word discovery, in: Proceedings of the Interspeech, 2008, pp. 2570-2573.

[24] E.L. Newport, Statistical language learning in human infants and adults, in: Proceedings of the Interspeech, Pittsburgh, USA, 2006.

[25] E. Gaussier, C. Goutte, Relation between PLSA and NMF and implications, in: Proceedings of the SIGIR, Salvador, Brazil, 2005.

[26] T. Hoffman, Probabilistic latent semantic analysis, in: Proceedings of Uncertainty in Artificial Intelligence, Stockholm, 1999.

[27] H. Van hamme, HAC-models: a novel approach to continuous speech recognition, in: Proceedings of the Interspeech, Brisbane, Australia, 2008.

[28] H. Van hamme, Integration of asynchronous knowledge sources in a nove speech recognition framework, in: Proceedings of the ITRW on Speech Analysis and Processing for Knowledge Discovery, Aalborg, Denmark, 2008.

[29] L. Boves, L. ten Bosch, R. Moore, ACORNS - towards computational modelling of communication and recognition skills, in: Proceedings of the ICCI, California, USA, 2007

[30] S.Z. Li, X. Hou, H. Zhang, Q.S. Cheng, Learning spatially localized, parts-based representation, in: Proceedings of the IEEE Computer Conference on Computer Vision and Pattern Recognition (CVPR), 2001, pp. 207-212.

[31] P.D. O'Grady, B.A. Pearlmutter, Convolutive non-negative matrix factorisation with a sparseness constraint, in: Proceedings of the IEEE International Workshop on Machine Learning for Signal Processing (MLSP), 2006, pp. 427-432.

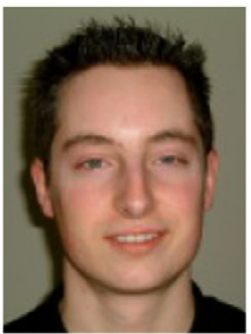

Joris Driesen received the master degree in electrical engineering (burgerlijk ingenieur) from the Katholieke Universiteit Leuven (KUL) in 2006. After a period of working as a software developer at MediaGeniX NG, he returned to the KUL as a PhD candidate in 2007. His current research interests are language acquisition, data mining and optimization.

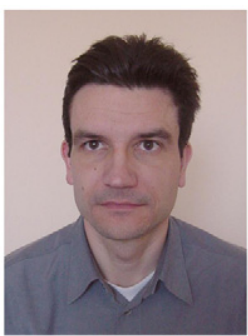

Hugo Van Hamme received the $\mathrm{PhD}$ degree in elec trical engineering from Vrije Universiteit Brussel (VUB) in 1992, the MSc degree from Imperial College, London, in 1988 and the Masters degree in engineering (burgerlijk ingenieur) from VUB in 1987. From 1993 til 2002, he worked for L\&H Speech Products and ScanSoft, initially as senior researcher and later as research manager. Since 2002, he is a professor at the department of electrical engineering of K.U.Leuven. His main research interests are: applications of speech technology in education and speech therapy, computationa models for speech recognition and language acquisition and noise robust speech recognition. 